\title{
Supplementary Information for Two-step crystal nucleation is selected because of lower surface free energy barrier
}

\author{
Maria Kaissaratos, Luis Filobelo, and Peter G. Vekilov \\ William A. Brookshire Department of Chemical and Biomolecular Engineering, University of \\ Houston, 4726 Calhoun Road, Houston, Texas 77204, USA.
}

\section{Methods}

\section{Solution preparation}

We used porcine insulin powder (Sigma-Aldrich) with no additional purification. The protein was dissolved in $0.02 \mathrm{M} \mathrm{HCl}$ (Fisher Scientific) to prepare stock solution. The stock was filtered through a $0.22 \mu \mathrm{m}$ pore size syringe filter (Millipore Millex-GV) and refrigerated at $4^{\circ} \mathrm{C}$ for later use. The insulin concentration of the stock was determined from the absorbance at wavelength $280 \mathrm{~nm}$ employing a Beckman DU 68 spectrophotometer and quartz cuvettes. The extinction coefficient assumed was $1.04 \mathrm{ml} \mathrm{mg}^{-1} \mathrm{~cm}^{-1}{ }^{1}{ }^{1}$ Detailed description of insulin solubility and concentration measurements can be found in Bergeron, et al. ${ }^{2}$

Before each experiment, a solution with a target concentration was prepared using the insulin stock. A protocol to grow 2-zinc rhombohedral insulin crystals was developed following Harding et al., ${ }^{3}$ Schlichtkrull, ${ }^{4-5}$ and McPherson. ${ }^{6}$ Zinc chloride (Sigma-Aldrich) was dissolved in water and the solution was clarified by adding $\mathrm{HCl}$ to a $\mathrm{pH}$ of ca. 3.23. We added this solution to an aliquot of insulin stock until $\mathrm{ZnCl}_{2}$ concertation reached 0.1 $\mathrm{M}$ We then added $0.2 \mathrm{M}$ tri-sodium citrate (Fisher); neat acetone (Fisher, HPLC-grade), if needed; and water to produce final concentrations of insulin $1-4 \mathrm{mg} \mathrm{ml}^{-1} ; \mathrm{HCl}, 0.01 \mathrm{M} ; \mathrm{ZnCl}_{2}, 0.005 \mathrm{M}$; Na citrate, $0.05 \mathrm{M}$; and acetone, $2.1 \mathrm{M}$. The final $\mathrm{pH}$ of the solutions was in the range $6.83-6.93$. In the experiments where acetone was not used, it was replaced with $0.02 \mathrm{M} \mathrm{HCl}$, which led to a $\mathrm{pH}$ lower by ca. 0.2 .

The solubility of insulin strongly depends on $\mathrm{pH}$. It has a minimum at $\mathrm{pH} 5.3$, which corresponds to the isoelectric point. ${ }^{7-8}$ The $\mathrm{pH}$ of the crystallizing system was measured at different temperatures to verify that it remained within the above range.

\section{Characterization of insulin purity}

Three different methods were used to characterize insulin purity: 1. Gel Phastgel Gradient 8-25 with native buffer strips (Pharmacia Biotech); 2. Phastgel High Density gel (Pharmacia Biotech). 3. Biosizing Protein 200 Plus Array chip (Agilent Technologies) with Agilent Technologies 2100 Bioanalyzer. For methods 1. and 2., we used current of $20.0 \mathrm{~mA}$, applied for $500 \mathrm{vh}$. The developed gels displayed a single band, indicating the absence of foreign material or monomer aggregation. Method 3. relies on SDS electrophoresis of fully denatured proteins. Hence, prior to testing, the insulin solution samples were held in a $100^{\circ} \mathrm{C}$ bath for four minutes. The results revealed mostly insulin monomers, and the presence of less than $4 \%$ contaminants, which likely originate from insulin biosynthesis in the pancreas, such as proinsulin and proinsulin intermediates. Whereas contaminants with molecular weight lower than $4.0 \mathrm{kDa}$ are beyond the reach of this method, they are smaller than insulin $\left(\mathrm{M}_{\mathrm{w}}=5.8 \mathrm{kDa}\right)$ and unlikely to affect insulin crystallization. ${ }^{9-10}$

\section{Characterization of the interactions in solution using static light scattering}


Static light scattering (SLS) experiments measured the second osmotic virial coefficient $\mathrm{B}_{2}$ of insulin and verified its molecular mass. We employed ALV light scattering device equipped with He-Ne laser $(\lambda=632.8 \mathrm{~nm}$, $35 \mathrm{~mW}$ ) and ALV-5000/EPP Multiple tau Digital Correlator (ALV-Gmbh, Langen, Germany). During the experiment, protein solutions were held in cylindrical cuvettes of volume from 0.5 to $1 \mathrm{ml}$ at $25^{\circ} \mathrm{C}$. Sample solutions were prepared and filtered into the cuvette immediately before each experiment. For a given sample concentration, three consecutive experiments were completed with a signal acquisition time of 20 seconds each. The tolerance of the variability of the count rate was limited to less than $2 \%$. The range of concentrations used was $0.67-1.80 \mathrm{mg} \mathrm{ml}^{-1}$ for samples containing $15 \%(\mathrm{v} / \mathrm{v})$ acetone and $0.28-2.01 \mathrm{mg} \mathrm{ml}^{-1}$ otherwise. The molecular mass of the hexamer, and the second osmotic virial coefficient in dimensional form were extracted from the data using Debye plots ${ }^{11-14}$ expressed by the scattering equation in the limit of low protein concentration $\mathrm{C}$

$$
\frac{K c}{R_{\theta}} M_{w}=1+2 B_{2} M_{w} C
$$

where $R_{\theta}$ is the Rayleigh ratio of the scattered to the incident light intensity, $B_{2}$ is the second virial coefficient (in $\mathrm{cm}^{-3} \mathrm{~mol} \mathrm{~g}^{2}$ ), $\mathrm{M}_{\mathrm{w}}$ is the molecular mass $\left(\mathrm{g} \mathrm{mol}^{-1}\right), \mathrm{K}$ is a system constant defined as

$$
K=\frac{1}{N_{A}}\left(\frac{2 \pi n_{o}}{\lambda^{2}}\right)^{2}\left(\frac{d n}{d C}\right)^{2},
$$

where $\mathrm{n}_{0}=1.331$ is the refractive index of the solvent at the wavelength of the laser $\lambda, \mathrm{N}_{\mathrm{A}}$ is Avogadro's number, and $\frac{\mathrm{dn}}{\mathrm{dC}}$ is the is the refractive index increment with protein concentration.

Determinations of the change of refractive index with respect to concentration, $\frac{\mathrm{dn}}{\mathrm{dC}}$, were performed using a Brookhaven differential refractometer operating at $\lambda=620 \mathrm{~nm}$ set to static mode. The equipment was turned on at least 12 hours before the beginning of the experiments to ensure temperature and voltage stability. Then it was washed with DI water and calibrated at $30^{\circ} \mathrm{C}$ using aqueous $\mathrm{KCl}$ standard solutions with concentrations 0.5 to $7 \mathrm{mg} \mathrm{ml}^{-1}$. We obtained differential refractive index for insulin solutions in $0.02 \mathrm{M} \mathrm{HCl}$ with no acetone $\mathrm{dn} / \mathrm{dC}$ $=0.2029 \pm 2.77 \times 10^{-3} \mathrm{~cm}^{3} \mathrm{~g}^{-1}$ (Fig. $5 \mathrm{~b}$ ). This result is very close to the value typical of numerous proteins, $0.2 \mathrm{~cm}^{3}$ $\mathrm{g}^{-1} \cdot{ }^{15-18}$ This similarity justifies the use of the same value $\left(\frac{d n}{d c}\right)$ for acetone-containing solutions, where the cosolvent destabilizes the signal and hampers the determination.

The molecular weight obtained from the Debye plots intercept at $\mathrm{C}=0$ was approximately $33,000 \mathrm{~g} \mathrm{~mol}^{-}$ 1. The difference with the published results could be accounted for by uncertainties of $\frac{\mathrm{dn}}{\mathrm{dC}}$ as well as small differences in the observed scattering volume of the protein sample and the toluene standard. We show Debye plots of insulin solutions in Fig. S1.

The values of the second osmotic virial coefficients were extracted from the slopes of the plots. For acetone-free insulin solutions $B_{2}=-6.70 \times 10^{-4} \mathrm{~cm}^{3} \mathrm{~mol} \mathrm{~g}^{2}$, and with acetone $B_{2}=-3.32 \times 10^{-3} \mathrm{~cm}^{3} \mathrm{~mol} \mathrm{~g}^{2}$.

\section{Insulin clusters count and size determination}

We employed Nanosight LM10-HS microscope (Nanosight Ltd) equipped with green laser $\lambda=532 \mathrm{~nm}$ and temperature control $\left(\mathrm{T}=22^{\circ} \mathrm{C}\right)$ to count and characterize individual clusters in insulin solutions in the presence and absence of acetone(Fig. 5a). ${ }^{15,19-25}$ The raw data of this method are movies of intensity patterns of clusters undergoing Brownian motion. The rate of movie acquisition depends on the camera characteristics and settings, in our experiments it was about 25 fps. We determined the clusters diffusivity by using a custom designed software package which analyzes individual cluster trajectories and calculates the mean squared displacement (Fig. 5b, c). The cluster radius $R$ was calculated using the Einstein-Stokes equation accounting for 
the previously determined viscosity. The scaling the number of clusters intensity patterns on each of frame with the observed volume produces cluster concentration. The result is displayed as a cluster size distribution (Fig. 5d).

\section{Experimental setup for nucleation rate determination}

The experimental method is based on temperature dependence of protein solubility, Fig. 2a. The protocol for the study of nucleation rate experiment required the protein solution to undergo two temperature stages. At the beginning of the experiment the temperature of the protein solution was set to $\mathrm{T}_{1}$, which took 15 30 seconds to quench from room temperature, so the nucleation process could be initiated at a desired supersaturation level. After a period of nucleation time $\Delta t$, the temperature was modified to a new value $T_{2}$, in 15 - 30 seconds, decreasing the thermodynamic supersaturation level, so new nuclei could not form, but the existing ones could grow to a visible size, Fig. $2 \mathrm{~b}$.

We used an experimental device similar to the one proposed by Galkin and Vekilov. ${ }^{26-30}$ Insulin solutions were loaded in five Teflon plates with individual temperature control in which a temperature and time allowed for nucleation could be controlled independently, Fig. 2 c. To account for the stochastic nature of the individual nucleation events, a total of 2000 samples were used for each experiment. Four hundred wells machined on each Teflon insert held the sample droplets separated from each other at all times. Each insert was pressed against a $6 \mathrm{~mm}$ brass plate, and was secured to it by a square-shaped brass frame and screws. A thin layer of Omegatherm 201 high thermal conductivity paste was placed in between the insert and the brass plate to ensure uniform heat transfer between them.

With the purpose of controlling the temperature of the samples, each brass plate was placed on top of a $35 \times 35 \mathrm{~mm}^{2}$ thermoelectric Peltier element model DT12-6-01S. Each Peltier element, along with a type K thermocouple, was connected to independent Marlow Industries temperature controllers (model number SE5010). The thermocouple was inserted into the brass plate to monitor the temperature constantly and to provide feedback to the temperature controller.

A computer linked to the five controllers regulated the temperature and the time at which the plates remained at such temperature. The five thermoelectric Peltier elements were mounted on a long aluminum plate to facilitate the heat removal, then looped back to a Neslab cooling water circulator model RTE-100. Although the whole range was never used, this arrangement allowed variations of the temperatures from -10 to $65^{\circ} \mathrm{C}$, which is the highest limit for the Peltier element.

The whole assembly was mounted on a computerized Aerotech Inc. XY translational stage (models ATS02040-M-02L and ATS03005-M) and placed under a Leica MZ8 microscope with a 2.0× objective. To get additional enhancement of the image, a 24-bit digital camera (Sony DKC-5000, 3CCD), with resolution of $1280 \times$ 1280 pixels ${ }^{2}$ was attached to the top of the microscope and connected to a video monitor.

The solution was dispensed on the Teflon inserts using a computer-controlled pump consisting of a gastight $2.5 \mathrm{ml}$ syringe (Hamilton Co., model 814420) mounted on an Aerotech mechanical stage model ATS03005M. To prevent unwanted crystallization in the syringe, the temperature of the solution in it was maintained constant using a water bath, forcing a low supersaturation. A Grant industries heating water circulator type $\mathrm{KD} / \mathrm{KT}(\mathrm{L})$ kept the water bath at a selected temperature.

The pump was calibrated using de-ionized water. The step size of the mechanical stage controller was set to a specific value, afterwards 100 droplets were deposited in a weighting boat. The size of a droplet for experiments was chosen $0.7 \mu \mathrm{l}$.

To verify that chosen $\mathrm{T}_{2}$ was appropriate (i.e. no new nuclei appeared during the time frame of the experiment), several temperatures in the range from $8^{\circ}$ to $26^{\circ} \mathrm{C}$ were tested. All five Teflon plates were loaded 
with identical protein solution each time, but were kept at different temperatures for 24 hours. After the growth period, the plates containing 200 droplets each was scrutinized under the microscope to count crystals formed. In order to reduce the error introduced by new nucleation during $\mathrm{T}_{2}$ to less $2 \%$, when more than four crystals were found on a plate, the tested temperature was rejected. At the end of the testing, $\mathrm{T}_{1}$ was selected at $13^{\circ} \mathrm{C}$ and $\mathrm{T}_{2}$ was set at $22^{\circ} \mathrm{C}$.

The chosen temperatures were tested in two different ways to verify that the nucleation was not limited by depletion of the crystallizing solution during $\Delta$ t. In the first test, it was observed that at the end of the nucleation period no crystals reached visible size in the plates, but they grew to sizes of 50-300 $\mu \mathrm{m}$ at the end of the $\mathrm{T}_{2}$ temperature stage, depending on the conditions of the experiment. This shows that only a few molecules were clustered in a few nuclei at the end of the nucleation period. During the second test, the nucleation setup completed a cycle of nucleation and a short period of growth. Under the microscope, a few crystals of about 50 $\mu \mathrm{m}$ were observed in the droplets. A second cycle of nucleation and growth followed afterwards, yielding new smaller crystals in the same droplets. This demonstrated that no depletion of solution occurred during the first cycle in spite of having large crystals already present.

\section{Determination of equilibrium concentration for the system buffer- acetone-silicon oil}

During the first attempts to grow insulin crystals using acetone as co-solvent, it was observed that the latter diffused out of the droplet samples into the surrounding silicon oil. This migration modified the acetone concentration in the crystallizing samples to unknown degrees, resulting in irreproducible conditions.

To gain control on the concentration of acetone in the buffer solution during the nucleation rate experiments, it was necessary to have acetone present in the surrounding silicon oil to achieve chemical equilibrium and stop the emigration of acetone out of the crystallizing samples. Since neither the silicon oil nor the buffer absorb light at a wavelength of $280 \mathrm{~nm}$, a calibration curve was prepared to determine the concentration of acetone in buffer solution. Several buffer-acetone and oil-acetone samples were prepared and combined to find the adequate proportions to obtain a concentration of $2.1 \mathrm{M}$ of acetone in the buffer once the chemical equilibrium had been reached. This was achieved by using a mixture of $3.2 \% \mathrm{vol} / \mathrm{vol}$ acetone in silicon oil.

\section{Data collection and reproducibility}

The insulin solutions prepared as discussed above produced perfect, well defined rhombohedral crystals with space groups R3. ${ }^{3}$ The nucleation process is intrinsically stochastic: the number of crystals that appear in a certain volume under identical conditions can be, for instance, one, three, or zero. The repetition of the same experiment yielded different number of crystals. To extract a nucleation rate from these samples, it is necessary to employ a large number of statistically independent experiments. The experimental distributions from all five Teflon inserts produced similar distributions and could be compared with the Poisson law ${ }^{28}$

$$
P(m)=\frac{N^{m}}{m !} \exp (-N)
$$

where $m$ is the number of crystals that appeared in volume $V$ during time $\Delta t$, and $N$ is the mean value of the distribution.

To evaluate the correspondence of the experimental data and the Poisson law, the parameter $\chi^{2}$ was calculated from

$$
\chi^{2}=\sum_{m} \frac{\left[F_{m}-P(m) N_{\text {trial }}\right]^{2}}{P(m) N_{\text {trial }}}
$$


where $F_{m}$ ar the measured frequencies, $P(m)$ are the Poissonian best-fit values, and $N_{\text {trial }}$ is the number of samples used to determine the mean value.

The levels of confidence obtained using this criterion were above $95 \%$ for most experiments, although some dropped to $88 \%$. The good correspondence of the experimental distributions and the Poisson law ensure that all individual nucleation events are independent from each other. Figures 1 (b-f) show the distributions obtained for insulin experiments. As the nucleation time was increased, the mean value of the distribution also increased. The continuous lines represent the Poisson fit.

The intrinsic error of such determinations (i.e. deviation of the calculated value from the actual value) is calculated from the ratio

$$
\text { error }=\sqrt{\frac{N}{N_{\text {trial }}}},
$$

Where $N$ is the mean value of the distribution, and $N_{\text {trial }}$ is the number of samples used to determine the mean value.

\section{Determination of the steady-state homogeneous nucleation rate}

The distribution of number of crystals in one droplet changes with nucleation time $\Delta t$ (Fig. 3b-f). The mean number of crystals nucleated increases linearly with time, suggesting steady state nucleation regime at the selected conditions (Fig. 3g).

Several factors could temper the steadiness of the system. Depletion caused by denaturation of protein, and bacteriological or enzymatic action might consume the protein in the samples. These competing processes have characteristic times comparable to crystal nucleation times, and they could produce reduced values of nucleation rates and a sublinear dependence $\mathrm{N}(\mathrm{t})$. The straight line in Figure $3 \mathrm{~g}$ indicates that none of these factors affected the experimental results. Another source of unsteadiness could be the depletion of monomers in the solution caused by the excessive nucleation. This issue was addressed by running a two-cycle experiment, showing that the solution was not depleted at the end of nucleation time $\Delta \mathrm{t}$.

Even though the samples are submerged in silicon oil to reduce heterogeneous nucleation on the interface of the droplets, it still occurs. The y-axis intercept of the evoutions of the mean number of nucleated crystals is greater than zero (Fig. $3 g$ ). It is likely that particles smaller than the filter pore size $(0.22 \mu \mathrm{m})$ serve as centers of heterogeneous nucleation. The good linearity of $\mathrm{N}(\mathrm{t})$ for $\mathrm{t}>0$ suggests that this happens at times shorter than those accessible to the technique. This fast nucleation adds a constant positive intercept in the linear dependence of the number of crystals nucleated at times $t>0$ as discussed by Galkin and Vekilov. ${ }^{26-28}$

A discrete value of the homogeneous nucleation rate was obtained for a specific concentration of protein utilizing a linear regression. The homogeneous nucleation rate is extracted from the slope applying

$$
J=\frac{\Delta N}{V \times \Delta t}=\frac{j}{V}
$$

where $j$ is the slope of the straight line and $V$ is the volume of one droplet.

A series of experiments were performed to obtain dependences of homogeneous nucleation rates as a function of protein concentration.

\section{Supplementary references}

1. Pace, C. N.; Vajdos, F.; Fee, L.; Grimsley, G.; Gray, T., How to measure and predict the molar absorption coefficient of a protein. Protein Science : A Publication of the Protein Society 1995, 4, 24112423. 
2. Bergeron, L.; Filobelo, L. F.; Galkin, O.; Vekilov, P. G., Thermodynamics of the Hydrophobicity in Crystallization of Insulin. Biophysical Journal 2003, 85, 3935-3942.

3. Harding, M. M.; Hodgkin, D. C.; Kennedy, A. F.; O'Conor, A.; Weitzmann, P. D., The crystal structure of insulin. II. An investigation of rhombohedral zinc insulin crystals and a report of other crystalline forms. J Mol Biol 1966, 16, 212-26.

4. Schlichtkrull, J., INSULIN CRYSTALS .1. THE MINIMUM MOLE-FRACTION OF METAL IN INSULIN CRYSTALS PREPARED WITH ZN++, CD++, $\mathrm{CO}++, \mathrm{NI++}, \mathrm{CU}++, \mathrm{MN++}$, OR FE++. Acta Chemica Scandinavica 1956, 10, 1455-1458.

5. Schlichtkrull, J., INSULIN CRYSTALS .2. SHAPE OF RHOMBOHEDRAL ZINC-INSULIN CRYSTALS IN RELATION TO SPECIES AND CRYSTALLIZATION MEDIA. Acta Chemica Scandinavica 1956, 10, 1459-1464.

6. McPherson, A., Crystallization of biological macromolecules. Cold Spring Harbor Laboratory Press: Cold Spring Harbor New York, 1999.

7. Fischel-Ghodsian, F.; Brown, L.; Mathiowitz, E.; Brandenburg, D.; Langer, R., Enzymatically controlled drug delivery. Proceedings of the National Academy of Sciences of the United States of America 1988, 85, 2403-2406.

8. Brange, J., Galenics if insulin: the physico-chemical and pharmaceutical aspects of insulin and insulin prearations. Springer: Berlin, 1987.

9. Vekilov, P. G., Molecular mechanisms of defect formation. In Methods in Enzymology, C.W.

Carter, J.; Sweet, R. M., Eds. Academic Press: San Diego, 2003; Vol. 368: Macromolecular

Crystallography, Part C, pp 170-188.

10. Yau, S.-T.; Thomas, B. R.; Galkin, O.; Gliko, O.; Vekilov, P. G., Molecular mechanisms of microheterogeneity-induced defect formation in ferritin crystallization. Proteins: Structure, Function, Genetics 2001, 43, 343-352.

11. Petsev, D. N.; Thomas, B. R.; Yau, S.; Vekilov, P. G., Interactions and aggregation of apoferritin molecules in solution: effects of added electrolytes. Biophysical Journal 2000, 78, 2060-2069.

12. Muschol, M.; Rosenberger, F., Interactions in undersaturated and supersaturated lysozyme solutions: Static and dynamic light scattering results. The Journal of Chemical Physics 1995, 103, 1042410432.

13. Petsev, D. N.; Vekilov, P. G., Evidence for Non-DLVO Hydration Interactions in Solutions of the Protein Apoferritin. Physical Review Letters 2000, 84, 1339-1342.

14. Petsev, D. N.; Chen, K.; Gliko, O.; Vekilov, P. G., Diffusion-limited kinetics of the solution-solid phase transition of molecular substances. Proceedings of the National Academy of Sciences 2003, 100, 792-796.

15. Vorontsova, M. A.; Chan, H. Y.; Lubchenko, V.; Vekilov, P G., Lack of Dependence of the Sizes of the Mesoscopic Protein Clusters on Electrostatics. Biophysical Journal 2015, 109, 1959-1968.

16. Petsev, D. N.; Wu, X.; Galkin, O.; Vekilov, P. G., Thermodynamic functions of concentrated protein solutions from phase equilibria. J. Phys. Chem. B 2003, 107, 3921-3926.

17. Petsev, D. N.; Chen, K.; Gliko, O.; Vekilov, P. G., Diffusion-limited kinetics of the solution-solid phase transition of molecular substances. Proc. Natl. Acad. Sci. USA 2003, 100, 792-796.

18. Feeling-Taylor, A. R.; Yau, S.-T.; Petsev, D. N.; Nagel, R. L.; Hirsch, R. E.; Vekilov, P. G., Crystallization Mechanisms of Hemoglobin C in the R State. Biophys. J. 2004, 87, 2621-2629.

19. Yang, D. S.; Saeedi, A.; Davtyan, A.; Fathi, M.; Sherman, M. B.; Safari, M. S.; Klindziuk, A.; Barton, M. C.; Varadarajan, N.; Kolomeisky, A. B.; Vekilov, P. G., Mesoscopic protein-rich clusters host the nucleation of mutant p53 amyloid fibrils. Proceedings of the National Academy of Sciences 2021, 118, e2015618118.

20. Warzecha, M.; Safari, M. S.; Florence, A. J.; Vekilov, P. G., Mesoscopic Solute-Rich Clusters in Olanzapine Solutions. Crystal Growth \& Design 2017, 17, 6668-6676. 
21. Safari, M. S.; Poling-Skutvik, R.; Vekilov, P. G.; Conrad, J. C., Differential dynamic microscopy of bidisperse colloidal suspensions. npj Microgravity 2017, 3, 21.

22. Safari, M. S.; Byington, M. C.; Conrad, J. C.; Vekilov, P. G., Polymorphism of Lysozyme Condensates. The Journal of Physical Chemistry B 2017, 121, 9091-9101.

23. Vorontsova, M. A.; Vekilov, P. G.; Maes, D., Characterization of the diffusive dynamics of particles with time-dependent asymmetric microscopy intensity profiles. Soft Matter 2016, 12, 69266936.

24. Byington, M. C.; Safari, M. S.; Conrad, J. C.; Vekilov, P. G., Protein Conformational Flexibility Enables the Formation of Dense Liquid Clusters: Tests Using Solution Shear. The Journal of Physical Chemistry Letters 2016, 7, 2339-2345.

25. Li, Y.; Lubchenko, V.; Vekilov, P. G., The Use of Dynamic Light Scattering and Brownian Microscopy to Characterize Protein Aggregation. Rev. Sci. Instrum. 2011, 82, 053106

26. Galkin, O.; Vekilov, P. G., Control of protein crystal nucleation around the metastable liquidliquid phase boundary. Proc. Natl. Acad. Sci. USA 2000, 97, 6277-6281.

27. Galkin, O.; Vekilov, P. G., Are Nucleation Kinetics of Protein Crystals Similar to Those of Liquid Droplets? Journal of the American Chemical Society 2000, 122, 156-163.

28. Galkin, O.; Vekilov, P. G., Direct Determination of the Nucleation Rates of Protein Crystals. The Journal of Physical Chemistry B 1999, 103, 10965-10971.

29. Vekilov, P. G.; Galkin, O., On the methods of determination of homogeneous nucleation rates of protein crystals. Colloids and Surfaces A: Physicochemical and Engineering Aspects 2003, 215, 125-130. 30. Filobelo, L. F.; Galkin, O.; Vekilov, P. G., Spinodal for the solution-to-crystal phase transformation. J. Chem. Phys. 2005, 123, 014904. 


\section{Supplementary Figures}

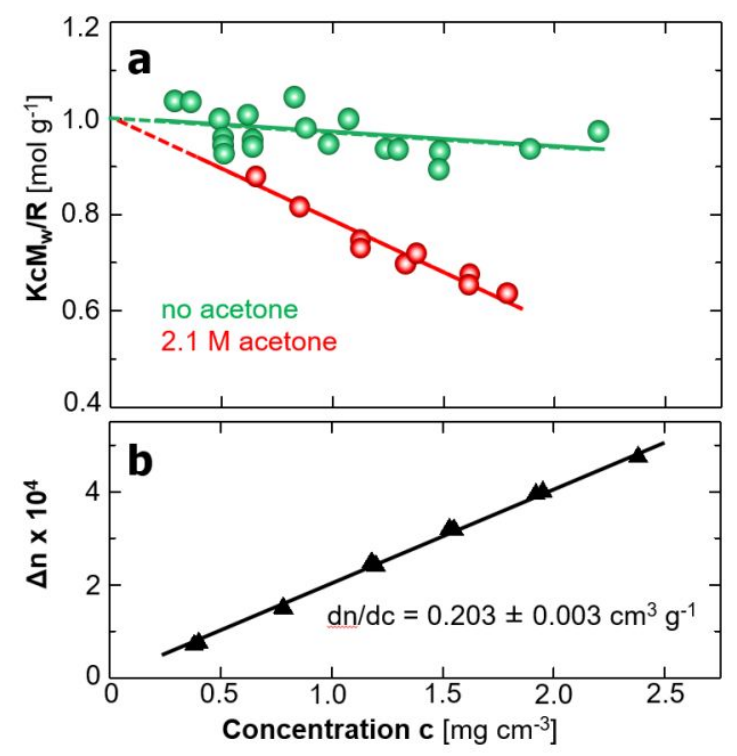

Fig. S1. Characterization of the intermolecular interactions and determination of the second osmotic virial coefficient $B_{2}$. (a) Debye plots of the intensity of light scattered from insulin solutions $K C / R(C)$ in the presence of $2.1 \mathrm{M}$ and in the absence of acetone. Straight lines represent the least square fit of the data. (b) The correlation between the refractive index increment $\Delta \mathrm{n}$ and $\mathrm{c}$ in solutions without acetone. The contributions of the acetone to $\mathrm{dn} / \mathrm{dC}$ prevented accurate measurements of $\Delta \mathrm{n}$ when acetone was added. 